\title{
Study on the vibration damping properties of the damping material applying rubber compounding magnetic powder (DRM)
}

\author{
Masanori Hansaka and Naoto Mifune \\ Railway Technical Research Institute of Japan, \\ 2-8-38, Hikari-cho, Kokubunji, 185 Japan
}

(Received 29 November 1996)

\begin{abstract}
Use of high grade damping materials is one of the effective methods to reduce vibration and noise, but the conventional damping materials have two weighty drawbacks, one is that it takes much labor to install, and the other is that it exhibits high damping performance at limited frequencies and temperatures. To overcome these drawbacks, we have developed a new type damping material Damping Material Applying Rubber Compounding Magnetic Powder (DRM). By compounding magnetic powder, DRM has not only a good workability but also enhanced damping abilities. As the result of experiments, the following properties are revealed. (1) The damping performance of DRM has a peak value on the magnetic attractive force. (2) The damping performance of DRM increases with a decrease in the ferrite content. (3) The damping performance of DRM increases with an increase in the vibratory amplitude while the conventional constraining type damping material has very little dependency on the vibratory amplitude. These properties are produced by the frictional loss generated between DRM and the vibrating body.
\end{abstract}

Keywords: Railway, Magnetism, Butyl rubber, Damping material, Noise and vibration

PACS number: 43. 40. Vn

\section{INTRODUCTION}

Use of high grade damping material is one of the effective methods to reduce vibration and noise. ${ }^{1,2)}$ With an increasing need for the vibration and noise control, the importance for the vibrating damping materials is mounting. But the conventional damping materials have two weighty drawbacks, one is that it takes much labor to install, and the other is that it can exhibit high damping performance only at limited frequencies and temperatures. To overcome these drawbacks, we have developed a new type damping material, Damping Material Applying Rubber Compounding Magnetic Powder, which is named DRM. ${ }^{3-6)}$ DRM, an application of the rubber compounding magnetic powder, has not only a good workability but also superior damping abilities. The damping performance of DRM is likely to be influenced strongly by the following factors; magnetic attractive force, ferrite content, and vibratory amplitude of vibrating body. As a result of examining the influences of these factors, the damping properties of DRM are made clear.

\section{DAMPING MECHANISM OF DRM}

The structure of DRM is shown in Fig. 1. DRM consists of two layers, namely, a magnetic rubber layer which is a mixture of ferrite-magnetizing powder and a constraining layer which is made of a highly rigid material, for example, steel, plaster, ceramics, and so on. DRM is easily attracted to a vibrating steel body by the magnetic attractive force. Damping mechanism of DRM is shown in Fig. 2. When DRM is vibrating with a vibrating steel body, a sliding friction is generated on the interface between the magnetic rubber layer and the vibrating 


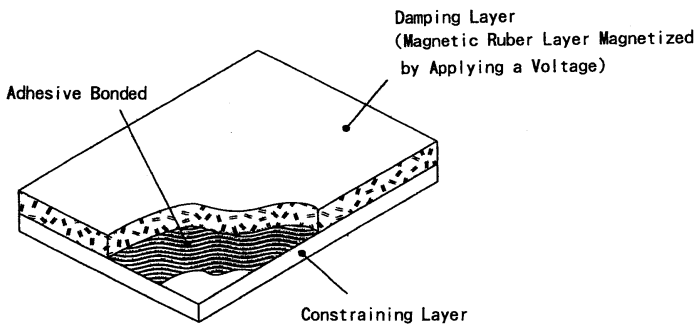

Fig. 1 Structure of DRM (damping material applying rubber compounding magnetic powder).

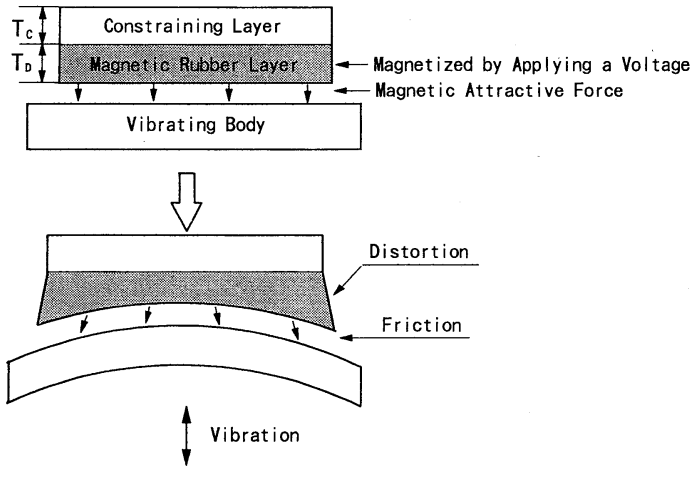

Fig. 2 Damping mechanism of DRM (damping material applying rubber compounding magnetic powder).

body. The sliding friction has an effect of dissipating a portion of vibration energy as thermal energy. This frictional loss acts together with the internal loss caused by the deformation of the magnetic rubber layer, and exhibits prominent damping effects.

\section{EXPERIMENTAL}

3.1 Factors Influencing the Damping Properties of DRM

Main factors which give decisive influence are as follows.

- Magnetic attractive force.

- Ferrite content.

- Dynamic coefficient of friction between DRM and a steel body (vibrating body).

- Vibratory amplitude of the vibrating body.

To make clear the damping properties of DRM, we investigated the influences of these factors.

\subsection{Samples}

Three kinds of measurements were carried out, namely, measurements of $\eta, \mu$ and $F$. $\eta$, that is a loss factor of DRM, is a representative index showing the damping performance of damping material. $\mu$ is a dynamic coefficient of friction between DRM and a steel body. $F$ is a magnetic attractive force per unit area of DRM. Through all cases of measurements, the sample was the following type of DRM, whose magnetic rubber layer was made of butyl rubber and constraining layer was hydrated calcium silicate. The magnetic rubber layer was magnetized by applying a voltage. The voltage was in the range from $700 \mathrm{~V}$ to $1.7 \mathrm{kV}$. The size of sample was $10 \times 200 \times$ sample thickness $(\mathrm{mm})$ for the measurement of $\eta, 40 \times 40 \times$ thickness $(\mathrm{mm})$ for that of $\mu$ and $F$. The thickness of the constraining layer $T_{\mathrm{C}}$ was $14 \mathrm{~mm}$ and the thickness of the magnetic rubber layer $T_{\mathrm{D}}$ was $3 \mathrm{~mm}$ through all cases of measurements. The mass of sample was $65 \mathrm{~g}$ for the measurement of $\eta, 45 \mathrm{~g}$ for that of $\mu$ and $F$. The vibrating body was a steel plate the size of $10 \mathrm{~mm}$ width $\times 220 \mathrm{~mm}$ length (from the fixed side to the free end) $\times 2.3 \mathrm{~mm}$ thickness. In the measurement of $\eta$, DRM was vibrating with the vibrating body. Two methods were selected to hold DRM to the vibrating body, one was to attach DRM by the magnetic attractive force and the other by adhesive bonding. The damping performance of magnetically attracted DRM is caused by both the frictional loss and the internal loss, while that of adhesive bonded DRM is only by the internal loss. By comparing two results, the damping properties of DRM characterized by the frictional loss are made clear. Since DRM is magnetically attracted to the vibrating body in practical use, in this paper, the damping performance of DRM means that of the magnetically attracted sample and the adhesive bonded sample is named AB-DRM for short.

\subsection{Experimental Method}

a. Loss factor $\eta$

Measuring instrument for $\eta$ is shown in Fig. 3. $\eta$ was measured by a cantilever type specimen. One side of the vibrating body was fixed and the free end was vibrated by an electromagnetic exciter. A measured spectrum of vibratory mobility of the vibrating body with DRM is shown in Fig. 4 . We found an apparent resonant peak to be around 400 Hz. We estimated $\eta$ at this peak by Eq. (1).

$$
\eta=\Delta f / f_{0}
$$




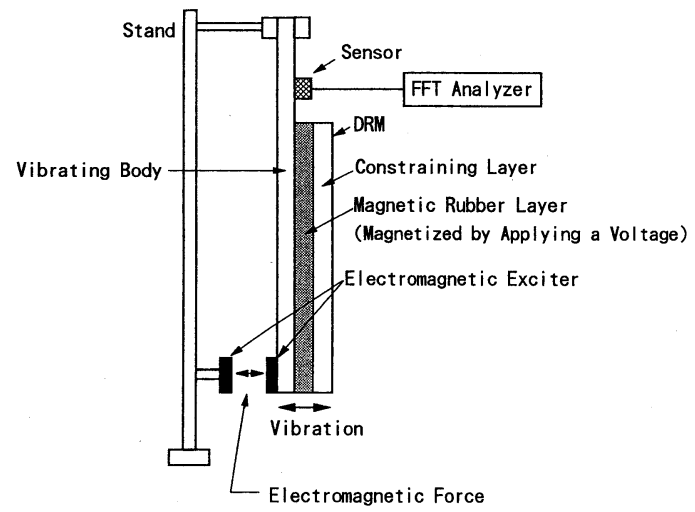

Fig. 3 Measuring instrument for loss factor.

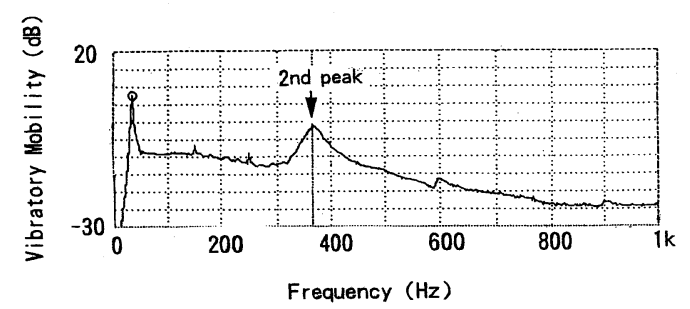

Fig. 4 Spectrum of vibratory mobility of steel body with DRM.

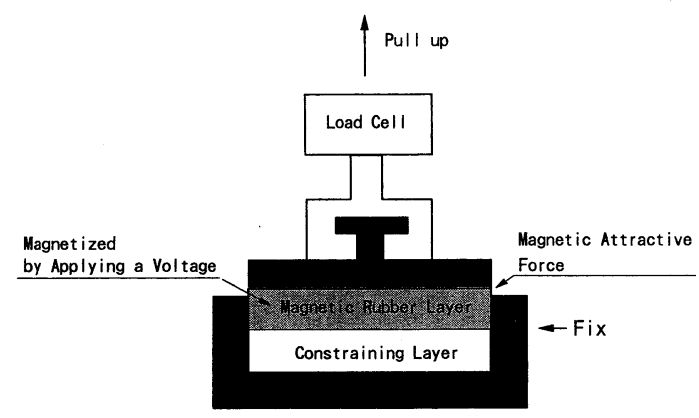

Fig. 5 Measuring instrument for magnetic attractive force.

Where $f_{0}$ is a resonant frequency $(\mathrm{Hz}), \Delta f$ is a half-value bandwidth at $f_{0}(\mathrm{~Hz})$.

b. Magnetic attractive force per unit area $F$

Measuring instrument for the magnetic attractive force of DRM is shown in Fig. 5. Surface of the constraining layer of DRM was fixed and surface of the magnetic rubber layer was attracted to a steel plate. The steel plate was pulled up by a tensile machine equipped with a load cell at a rate of 1 $\mathrm{mm} / \mathrm{min}$, and the maximum load at which the steel plate was pulled off was measured. $F$ was calcu-

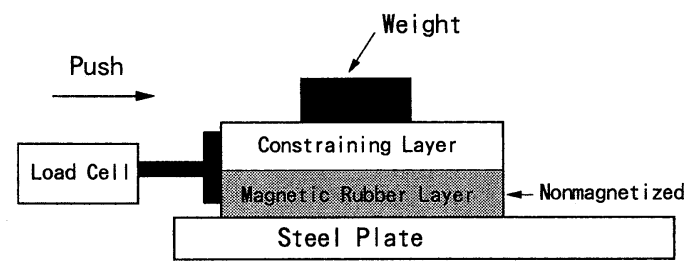

Fig. 6 Mesuring instrument for dynamic coefficient of friction between DRM and steel plate.

lated by dividing the measured load by the surface area of the magnetic rubber layer.

c. Dynamic coefficient of friction between DRM and a steel body $\mu$

Measuring instrument for $\mu$ is shown in Fig. 6 . A nonmagnetized DRM was placed on a steel plate with a weight of $174 \mathrm{~g}$, and it was pushed on the center of the side manually and horizontally with a load cell. The load was measured when DRM moved at even rate of about $50 \mathrm{~mm} / \mathrm{min}$, and $\mu$ was estimated by Eq. (2).

$$
\mu=l f / m g
$$

Where $l f$ is a measured load (N) and $m$ is a mass of the weight and DRM (215 g) and $g$ is the gravitational acceleration $\left(9.8 \mathrm{~m} / \mathrm{s}^{2}\right)$.

More than five data were taken and averaged. All experiments were done at room temperature $\left(23 \pm 3^{\circ} \mathrm{C}\right)$.

\section{DAMPING PROPERTIES OF DRM}

\subsection{F (Magnetic Attractive Force per Unit Area)- Dependency}

$F, V$-dependencies of the loss factor $\eta$ are shown in Fig. 7. $F$ means the magnetic attractive force per unit area of DRM, V means the ferrite content. $\eta$ of DRMs with $V$ of $40,45,50,55$ and 60 volume\% were measured. $F$ of each DRM was changed by varying the magnetizing voltage. $F$ was obtained in the range from $F_{\mathrm{MIN}}$ to $F_{\mathrm{MAX}}$. Where $F_{\mathrm{MIN}}$ is the least value at which DRM was kept being attracted to the vibrating body, and $F_{\mathrm{MAX}}$ is the value of being fully magnetized. The correlation between the magnetizing voltage and $F$ is shown in Table 1 .

$\eta$ of AB-DRM, that is DRM attached to the vibration body by adhesive bonding, of each $V$ were plotted on the right $Y$-coordinate. According to Fig. 7, it is revealed that $\eta$ of DRM is apparently greater than that of AB-DRM in sample of each $V$. 


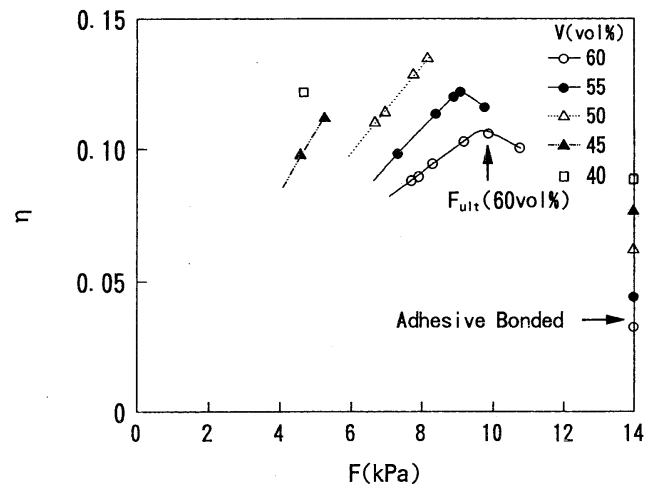

Fig. $7 \quad F$ and $V$-dependencies of loss factor $\eta$ of DRMs. $F$ : magnetic attractive force per unit area, $V$ : ferrite content.

Table 1 The correlation between magnetizing voltage and $F$.

\begin{tabular}{rccccc}
\hline \multirow{2}{*}{$V_{\mathrm{M}}(\mathrm{V})$} & \multicolumn{5}{c}{$F(\mathrm{kPa})$} \\
\cline { 2 - 6 } & 60 vol\% & $55 \mathrm{vol} \%$ & $50 \mathrm{vol} \%$ & $45 \mathrm{vol} \%$ & $40 \mathrm{vol} \%$ \\
\hline 1,700 & 10.8 & - & - & - & - \\
1,500 & 9.9 & 9.8 & - & - & - \\
1,300 & 9.2 & 9.1 & 8.2 & - & - \\
1,100 & 8.3 & 8.9 & 7.8 & - & - \\
900 & 7.9 & 8.4 & 7.0 & 5.3 & 4.7 \\
700 & 7.7 & 7.1 & 6.7 & 4.6 & - \\
\hline
\end{tabular}

$V_{\mathrm{M}}$ : Magnetizing voltage, $F$ : Magtetic attractive force per unit area

For example, $\eta$ of DRM, whose $V$ is 60 vol\% and $F$ is $10.8 \mathrm{kPa}$, is about one third greater than that of AB-DRM of 60 vol\%. The reason is likely to be that DRM has a great frictional loss in addition to an internal loss, while AB-DRM scarcely has the frictional loss.

From Fig. 7, it can be seen that $\eta$ of DRMs of 55 vol\% and 60 vol\% have a peak value on $F$, and $\eta$ of DRMs of 40 vol\%, $45 \mathrm{vol} \%$ and 50 vol\% improve simply with an increase of $F$. Here the ultimate value of $F$, which gives the peak value of $\eta$, is defined as $F_{\text {ult }} . \quad F_{\text {ult }}$ is found to be about $9.1 \mathrm{kPa}$ in sample of $55 \mathrm{vol} \%$ and about $9.9 \mathrm{kPa}$ in sample of 60 vol\%. From these results it follows that the damping performance of DRM has essentially a peak value on $F$, and the reason why no peak appears in DRMs of 40,45 and 50 vol\% is likely to be that $F$ of these DRMs don't reach $F_{\text {ult }}$ even when they are fully magnetized. These $F$-dependencies of the damping performance of DRM seem to originate from the $F$-dependency of the frictional loss. The reason why the frictional loss has a peak value is explained as follows. The frictional loss is given by a ratio of the energy loss dissipated by the frictional resistance to the energy of the vibration of the composite plate, that is a vibrating body with DRM. The energy loss caused by the frictional resistance corresponds to a work load consumed by the frictional resistance and it is given by a product of the frictional resistance and the sliding amplitude of DRM on the vibrating body. Consequently the frictional loss is expressed by Eq. (3).

$$
\eta_{\mathrm{f}}=\left(F_{\mathrm{R}} \times A_{\mathrm{s}}\right) / W
$$

Where $\eta_{\mathrm{f}}$ is a frictional loss, $F_{\mathrm{R}}$ is a frictional resistance (N) and $A_{\mathrm{S}}$ is a sliding amplitude of DRM on the vibration body $(\mathrm{m}), W$ is an energy of vibration of the composite plate $(\mathrm{J})$.

In the small range of $F, F_{\mathrm{R}}$ increases simply with an increase of $F$, whereas $A_{\mathrm{S}}$ isn't decisively influenced by $F$ but by the vibratory amplitude of the vibrating body because DRM slides without being braked by $F_{\mathrm{R}}$. Moreover $W$ changes little with a change in $F$ because the vibration amplitude of the composite plate is scarcely influenced by $F$. Reflecting these conditions, $\eta_{\mathrm{f}}$ improves simply with an increase of $F$ below $F_{\text {ult }}$. In the range of $F$ with excess intensity, $A_{\mathrm{S}}$ is damped because DRM is attracted strongly enough to be braked and the sliding of DRM on the interface of vibrating body is restrained. ${ }^{7)}$ Influenced by the decrease of $A_{\mathrm{S}}$, the frictional loss breaks down. Considering these assumptions, a standard expression of the damping performance of DRM is shown in Fig. 8. The damping performance of DRM is given by a sum of the internal loss and the frictional loss. The inter-

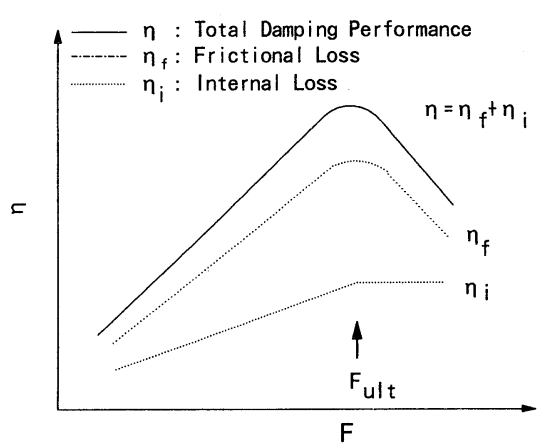

Fig. 8 Standard expression of damping performance of DRM. $F$ : magnetic attractive force per unit area, $F_{\text {ult }}$ : ultimate value of $F$. 


\section{HANSAKA and N. MIFUNE : STUDY ON THE DAMPING PROPERTIES OF DRM}

nal loss increases almost linearly with an increase of $F$ in its weak range, and gradually converges to a certain value. The frictional loss has a peak value on $F$, and ultimately the damping performance of DRM has a peak value on $F$. Under this assumption, the damping performance $\eta$ of DRM is given by Eqs. from (3) to (7) below $F_{\text {ult }}$.

$$
\begin{gathered}
\eta_{\mathrm{f}}=\left(F_{\mathrm{R}} \times A_{\mathrm{s}}\right) / W \\
F_{\mathrm{R}}=k \times F, \quad A_{\mathrm{s}}=u \\
\eta_{\mathrm{f}}=(k \times u \times F) / W=k_{\mathrm{1}} \times u \times F=a_{\mathrm{f}} \times F \\
\eta_{\mathrm{i}}=\eta_{\mathrm{ic}} / F_{\text {ult }} \times F=a_{\mathrm{i}} \times F \\
\eta=\eta_{\mathrm{f}}+\eta_{\mathrm{i}}=a_{\mathrm{f}} \times F+a_{\mathrm{i}} \times F=a \times F
\end{gathered}
$$

Where $\eta_{\mathrm{f}}$ is a frictional loss, $\eta_{\mathrm{i}}$ is an internal loss, $\eta_{\text {ic }}$ is a convergent value of $\eta_{\mathrm{i}}, u$ is a sliding amplitude of DRM on the vibrating body (constant for $F$ ) (m), $k, k_{1}, a, a_{\mathrm{f}}$ and $a_{\mathrm{i}}$ are constants.

Here Eq. (6) is based on an assumption that $F_{\text {ult }}$ is so strong that the deformation of the magnetic rubber layer at $F_{\mathrm{ult}}$ is as great as that of AB-DRM and $\eta_{\mathrm{i}}$ converges to the value of AB-DRM around $F_{\text {ult }}$.

Above $F_{\mathrm{ult}}$, representing the decrease of $A_{\mathrm{S}}$ with $\Delta u$, the frictional loss of DRM is given by the following equations.

$$
\begin{gathered}
\eta_{\mathrm{f}}=\left(F_{\mathrm{R}} \times A_{\mathrm{s}}\right) / W \\
F_{\mathrm{R}}=k_{\mathrm{l}} \times F=k_{1} \times\left(F_{\mathrm{ult}}+\Delta F\right), \quad A_{\mathrm{s}}=u-\Delta u \\
\eta_{\mathrm{f}}=\eta_{\mathrm{fp}}+k_{1} \times u \times \Delta F-k_{\mathrm{l}} \times F_{\mathrm{ult}} \times \Delta u \\
-k_{1} \times \Delta F \times \Delta u
\end{gathered}
$$

Where $\Delta F$ is $F-F_{\text {ult }}(\mathrm{kPa}), \eta_{\mathrm{fp}}$ is $\eta_{\mathrm{f}}$ at $F_{\mathrm{ult}}$ which is given by the term of $k_{1} \times F_{\mathrm{ult}} \times u$.

Here $u$ and $F_{\text {ult }}$ are constant for $F . \Delta u$ is assumed to increase linearly with an increase of $\Delta F$ because $\Delta u$ is generated by the effect of braking of $F_{\mathrm{R}}$. Consequently Eq. (9) is modified to equation (10).

$$
\begin{aligned}
\eta_{\mathrm{f}}= & \eta_{\mathrm{fp}}+\left(k_{1} \times u-k_{1} \times k_{2} \times F_{\mathrm{ult}}\right) \times \Delta F \\
& -k_{1} \times k_{2} \times(\Delta F)^{2}
\end{aligned}
$$

Where $k_{2}$ is a constant.

Under the assumption that $\eta_{\mathrm{i}}$ converges to a value at $F_{\text {ult }}$, the damping performance of DRM is given by Eq. (11) above $F_{\text {ult }}$.

$$
\begin{aligned}
\eta= & \left(k_{1} \times u-k_{1} \times k_{2} \times F_{\text {ult }}\right) \times \Delta F \\
& -k_{1} \times k_{2} \times(\Delta F)^{2}+\eta_{\mathrm{fp}}+\eta_{\mathrm{ic}} \\
= & \left(k_{1} \times u-k_{1} \times k_{2} \times F_{\text {ult }}\right) \times \Delta F \\
& -k_{1} \times k_{2} \times(\Delta F)^{2}+\eta_{\mathrm{p}}
\end{aligned}
$$

Where $\eta_{\mathrm{p}}$ is $\eta$ at $F_{\mathrm{ult}}$.

\subsection{V (Ferrite Content)-Dependency}

Turning to Fig. 7, it can be seen that the damping performance of DRM has apparent $V$-dependency on both DRM and AB-DRM. Reflecting these experimental results and Eq. (7), $a_{\mathrm{f}}, a_{\mathrm{i}}$ and a should be described as functions of $V$ and Eq. (7) is modified to Eq. (12).

$$
\begin{aligned}
\eta & =\eta_{\mathrm{f}}+\eta_{\mathrm{i}} \\
& =a_{\mathrm{f}}(V) \times F+a_{\mathrm{l}}(V) \times F=a(V) \times F
\end{aligned}
$$

The estimated values of $a_{\mathrm{f}}(V), a_{\mathrm{i}}(V)$ and $a(V)$ are shown in Fig. 9. According to Fig. 9 it is recognized that the internal loss increases almost linearly in our experimental range (from 40 vol\% to 60 vol\%) and the frictional loss increases exponentially with a decrease of $V$. The internal loss increases with an increase of rubber component. ${ }^{8)}$ The increase of the frictional loss is inferred to be brought about by the increase of $\mu$ because $F_{\mathrm{R}}$ is given by a product of $\mu$ and $F . V$ dependency of $\mu$ is shown in Fig. 10. As shown in Fig. $10, \mu$ has $V$-dependencies similar to $a_{\mathrm{f}}(V)$. The estimated value $b$ are shown in Fig. 11 and Table 2. Where $b$ is defined as $a_{\mathrm{f}}(V) / \mu(V)$. From Fig. 11 and Table 2, it is revealed that $b$ is almost constant for $V$. From these results it fol-

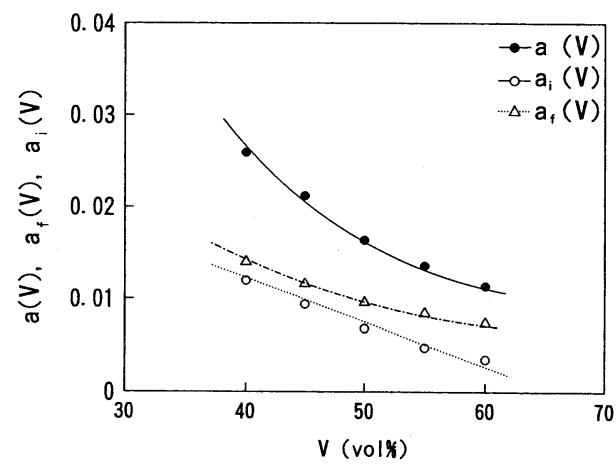

Fig. 9 Estimated values of functions $a(V)$, $a_{\mathrm{f}}(V), a_{\mathrm{i}}(V) . \quad \eta=\eta_{\mathrm{f}}+\eta_{\mathrm{i}}, \eta=a(V) \times F, \eta_{\mathrm{f}}=$ $a_{\mathrm{f}}(V) \times F, \quad \eta_{\mathrm{i}}=a_{\mathrm{i}}(V) \times F_{\mathrm{ult}} \quad\left(F \leqq F_{\mathrm{ult}}\right), \quad \eta$ : damping performance of DRM, $\eta_{\mathrm{f}}$ : frictional loss, $\eta_{\mathrm{i}}$ : internal loss. 
lows that $a_{\mathrm{f}}(V)$ is in proportional to $\mu(V)$ and the damping performance of DRM is formulated by Eq. (14) below $F_{\text {ult }}$.

$$
\begin{aligned}
& \left.\begin{array}{l}
a_{\mathrm{f}}(V)=k_{1} \times u=b \times \mu(V), \\
a_{\mathrm{i}}(V)=-c \times V+d
\end{array}\right\} \\
& \eta=\eta_{\mathrm{f}}+\eta_{\mathrm{i}}=a_{\mathrm{f}} \times F+a_{\mathrm{i}} \times F \\
& =b \times \mu(V) \times F+(-c \times V+d) \times F
\end{aligned}
$$

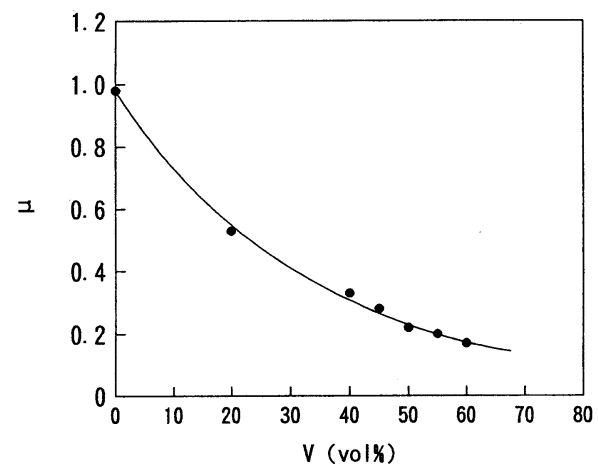

Fig. $10 V$-dependency of dynamic coefficient of friction $\mu$ between DRM and steel plate.

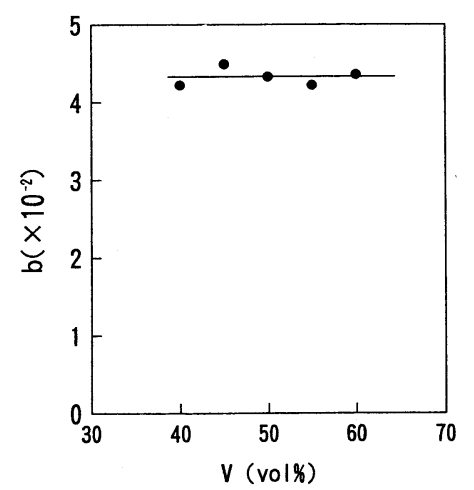

Fig. $11 V$-dependency of estimated value $b$. $\eta_{\mathrm{f}}=b \times \mu \times F \quad\left(F \leqq F_{\mathrm{ult}}\right), \quad \eta_{\mathrm{f}}$ : frictional loss, $F_{\text {ult }}$ : ultimate value of $F$.
Where $b, c, d$ are constants, $b$ is about $4.3 \times 10^{-2}$ $(1 / \mathrm{kPa}), c$ is about $4.2 \times 10^{-4}(1 / \mathrm{kPa} / \mathrm{vol} \%)$, and $d$ is about $2.9 \times 10^{-2}(1 / \mathrm{kPa})$.

Moreover $\mu(V)$ can be expressed by Eq. (15) from the experimental results.

$$
\mu(V)=e \times \exp (-f \times V)
$$

Where $e$ and $f$ are constants, $e$ is about $0.98, f$ is about $2.9 \times 10^{-2}$ (1/vol\%).

Finally the damping performance of DRM is expressed by Eq. (16) below $F_{\text {ult }}$.

$$
\begin{aligned}
\eta= & (b \times e \times \exp (-f \times V)) \times F \\
& +(-c \times V+d) \times F
\end{aligned}
$$

Above $F_{\text {ult }}$, the frictional loss is given by Eq. (11). Considering that $u$ is almost independent of $\mu(V)$ and the term of $k_{1} \times u$ is proportional to $\mu(V)$ as shown in Eq. (13), $k_{1}$ is inferred to be proportional to $\mu(V)$.

$\Delta u$, which means the decrease of $A_{\mathrm{S}}$ above $F_{\mathrm{ult}}$, is assumed to have an increase nearly in proportion to $\mu(V)$ because the effect of $F_{\mathrm{R}}$ grows linearly with an increase of $\mu(V)$. Consequently the constant $k_{2}$, which is given by $\Delta u / \Delta F$, is inferred to be nearly proportional to $\mu(V)$. Taking these conditions into consideration, the damping performance of DRM is given by Eq. (17) above $F_{\text {ult }}$.

$$
\begin{aligned}
\eta= & \eta_{\mathrm{p}}+\left(l_{1} \times \mu(V)-l_{2} \times F_{\text {ult }} \times \mu(V)^{2}\right) \times \Delta F \\
& -l_{2} \times(\mu(V) \times \Delta F)^{2}
\end{aligned}
$$

Where $l_{1}$ and $l_{2}$ are constants.

From Fig. 7 it is seen that $F_{\text {ult }}$ of DRM of 60 vol\%, whose $\mu(V)$ is smaller than that of DRM of 55 vol\%, is higher than that of DRM of 55 vol\%. The reason is explained as follows. The ultimate value of $F_{\mathrm{R}}$, above which the effect of $F_{\mathrm{R}}$ is generated clearly and $A_{\mathrm{S}}$ is damped, is likely to be nearly constant. Reflecting that $F_{\mathrm{R}}$ is given by a product of $F$ and $\mu(V), F_{\text {ult }}$ is nearly proportional to $\mu(V)^{-1}$. From these experimental results and

Table 2 Estimated values $b, b_{1}, b_{2}$ and $b_{3}$.

\begin{tabular}{ccccc}
\hline $\begin{array}{c}\text { Ferrite content } \\
(\text { vol\% })\end{array}$ & $b\left(\times 10^{-2}\right)$ & $b_{1}\left(\times 10^{-2}\right)$ & $b_{2}\left(\times 10^{-1}\right)$ & $b_{3}\left(\times 10^{-2}\right)$ \\
\hline 40 & 4.22 & - & - & - \\
45 & 4.49 & - & - & - \\
50 & 4.33 & - & - & - \\
55 & 4.22 & -0.11 & 2.15 & 4.63 \\
60 & 4.36 & -0.05 & 2.18 & 4.70 \\
\hline
\end{tabular}




\section{HANSAKA and N. MIFUNE : STUDY ON THE DAMPING PROPERTIES OF DRM}

assumptions, $F_{\text {ult }}$ can be estimated by Eq. (18).

$$
F_{\text {ult }}=l_{3} \times \mu(V)^{-1}+l_{4}
$$

Where $l_{3}$ and $l_{4}$ are constants.

From the experimental results $l_{3}$ is estimated to be about $0.9(\mathrm{kPa})$ and $l_{4}$ to be about $4.6(\mathrm{kPa})$. Substituting Eq. (18) into Eq. (17), Eq. (19) is derived.

$$
\begin{aligned}
\eta= & \eta_{\mathrm{p}}+b_{1} \times \mu(V) \times \Delta F-b_{2} \times \mu(V)^{2} \times \Delta F \\
& -b_{3} \times(\mu(V) \times \Delta F)^{2}
\end{aligned}
$$

Where $b_{1}, b_{2}, b_{3}$ are constants given in Eq. (20).

$$
b_{1}=l_{1}-l_{2} \times l_{3}, \quad b_{2}=l_{2} \times l_{3}, \quad b_{3}=l_{2}
$$

The results of estimating these constants by substituting the measured values of $\eta$ of DRMs of 55 vol\% and 60 vol\% into Eq. (19) are shown in Table 2.

As mentioned above, $F$ has a strong correlation with $V$. The reason why $F$ isn't related with a function $V$ in this study is as follows. $F$ is variable by varying the magnetizing voltage, so $F$ can be given independently of $V$. Practically $F$ is regarded as an important factor to adjust the damping performance of DRM.

\subsection{A (Vibratory Amplitude of Vibrating Body)- Dependency}

$A$-dependencies of $\eta$ of DRM and AB-DRM with 55 vol\% are shown in Fig. 12. $A$ means the vibratory amplitude of the vibrating body at the resonant frequency around $400 \mathrm{~Hz}$. $A$ was changed by varying the vibrating force with $1,2,3,4 \times 10^{-3} \mathrm{~N}$, and it was estimated by integrating the measured vibratory velocity of motion once. According to

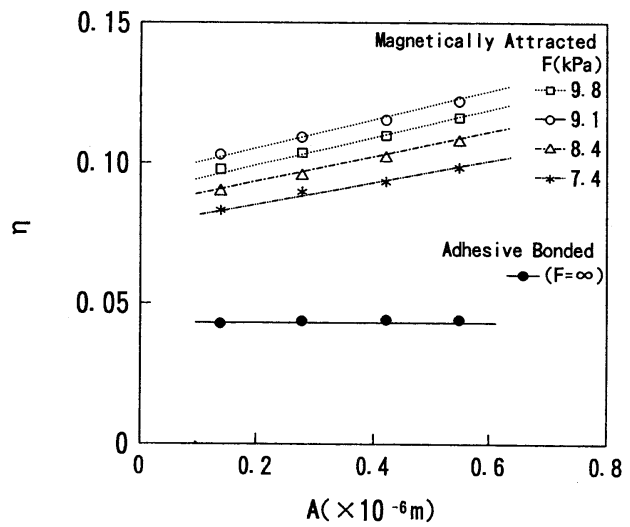

Fig. $12 A$-dependencies of loss factor $\eta$ of DRMs. $A$ : vibratory amplitude.
Fig. 12, it is found that $\eta$ of DRM increases apparently with an increase of $A$, whereas that of ABDRM changes little. The increase of the damping performance of DRM is owing to the increase of the frictional loss because $A_{\mathrm{S}}$ is promoted linearly with an increase of $A$. AB-DRM has little $A$ dependency due to the lack of the frictional loss.

Moreover from Fig. 12 it is shown that the damping performance of DRM linearly increases with an increase of $A$, so the damping performance of DRM can be formulated with Eq. (21).

$$
\eta=p \times A+q
$$

Where $p$ and $q$ are constants.

Comparing Eq. (21) with Eq. (14), $p, q$ should have clear dependencies on $F$ and $V . F$ and $V$-dependencies of the estimated value $p$ are shown in Fig. 13. From Fig. 13 it is recognized that $p$ has similar dependencies to those of $\eta$ of DRMs. Considering that $A$-dependency of $\eta$ is due to that of the frictional loss, the term of $p \times A$ should be linked with $\eta_{\mathrm{f}}$ and $q$ should be linked mainly with $\eta_{\mathrm{i}}$. Consequently Eq. (21) is modified to Eq. (22).

$$
\begin{aligned}
\eta= & p \times A+q \\
= & r \times \mu(V) \times F \times A+(-c \times V+d) \times F \\
& +\alpha(F, V)
\end{aligned}
$$

Where $r$ is a constant. $\alpha(F, V)$ is a function to match the experimental results with the calculated results by Eq. (22).

Comparing Eq. (22) with Eq. (14), $\alpha(F, V)$ proves to be expressed with the term of $s \times \mu(V) \times$ $F$, where $s$ is a constant. So Eq. (22) is modified to Eq. (23).

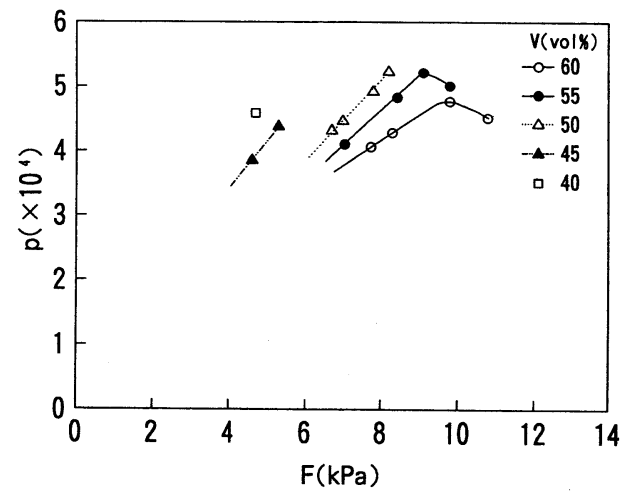

Fig. $13 \quad F$ and $V$-dependence of estimated value $p$. $p=\Delta \eta / \Delta A$. 
Table 3 Estimated values $r, s, r_{1}, s_{1}, s_{2}$ and $s_{3}$.

\begin{tabular}{ccccccc}
\hline $\begin{array}{c}\text { Ferrite content } \\
(\text { vol\%) }\end{array}$ & $r\left(\times 10^{4}\right)$ & $s\left(\times 10^{-2}\right)$ & $r_{1}\left(\times 10^{2}\right)$ & $s_{1}\left(\times 10^{-4}\right)$ & $s_{2}$ & $s_{3}$ \\
\hline 40 & 3.01 & 2.97 & - & - & - & - \\
45 & 2.96 & 2.90 & - & - & - & - \\
50 & 2.92 & 2.99 & - & - & - & - \\
55 & 2.90 & 2.81 & 7.56 & 7.32 & 5.1 & 1.1 \\
60 & 3.06 & 2.82 & 3.51 & 3.23 & 5.1 & 1.1 \\
\hline
\end{tabular}

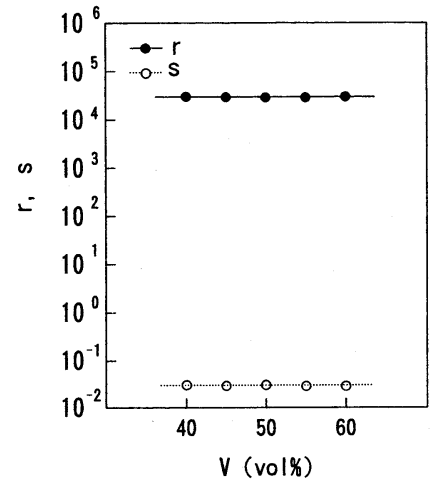

Fig. $14 V$-dependencies of estimated values $r$, s. $\quad \eta_{\mathrm{f}}=(r \times A+s) \times \mu \times F\left(F \leqq F_{\mathrm{ult}}\right), \eta_{\mathrm{f}}$ : frictional loss, $F_{\text {ult }}$ : ultimate value of $F$.

$$
\begin{aligned}
\eta= & (r \times A+s) \times \mu(V) \times F \\
& +(-c \times V+d) \times F
\end{aligned}
$$

The estimated values of $r$ and $s$ are shown in Fig. 14 and Table 3 . On average, $r$ is about $3.0 \times 10^{4}$ $(1 / \mathrm{m} / \mathrm{kPa})$ and $\mathrm{s}$ is about $2.9 \times 10^{-2}(1 / \mathrm{kPa})$.

Above $F_{\mathrm{ult}}$, as is the same with the case of below $F_{\text {uIt }}$, the frictional loss is assumed to be proportional to the term of $\left(r_{1} \times A+s_{1}\right)$ where $r_{1}$ and $s_{1}$ are constants. Taking this assumption into consideration, Eq. (19) is modified to Eq. (24).

$$
\begin{aligned}
\eta= & \left(r_{1} \times A+s_{1}\right) \times\left[\mu(V) \times \Delta F-s_{2} \times\left(\mu(V)^{2} \times \Delta F\right)\right. \\
& \left.-s_{3} \times(\mu(V) \times \Delta F)^{2}\right]+\eta_{\mathrm{p}}
\end{aligned}
$$

Where $r_{1}, s_{1}, s_{2}$ and $s_{3}$ are constants.

By substituting $F_{\text {ult }}$ for $F$ in Eq. (23), applying Eq. (18) and considering the internal loss to converge to a value at $F_{\mathrm{ult}}, \eta_{\mathrm{p}}$ is expressed by Eq. (25).

$$
\eta_{\mathrm{p}}=\left(r_{2} \times A+s_{4}\right) \times \mu(V)+r_{3} \times A+s_{5}+\eta_{\mathrm{ic}}
$$

Where $\eta_{\text {ic }}$ is the convergent value of $\eta_{\mathrm{i}}$ and $r_{2}, r_{3}$, $s_{4}, s_{5}$ are constants which are given in Eq. (26).

$$
r_{2}=r \times l_{4}, \quad r_{3}=r \times l_{3}, \quad s_{4}=s \times l_{4}, \quad s_{5}=s \times l_{3}
$$

Comparing the relationships between Eqs. (24) and (19) with that between Eqs. (23) and (14), the constants $r_{1}, s_{1}, s_{2}$ and $s_{3}$ are given by Eq. (27).

$$
r_{1}=r \times b_{1} / b, \quad s_{1}=s \times b_{1} / b, \quad s_{2}=b_{2} / b_{1}, \quad s_{3}=b_{3} / b_{1}
$$

The results of estimating these constants according to Eq. (27) are shown in Table 3. $\quad \eta$ calculated according to Eqs. (25) and (26) agree with the experimental data within an accuracy of $5 \%$.

\section{CONCLUSIONS}

The followings are the conclusions of this study on the damping properties of the Damping Material Applying Rubber Compounding Magnetic Powder, DRM.

(1) The damping performance of DRM magnetically attracted to the vibrating body is much enhanced compared with that of adhesive bonded DRM and it is shown how great is the role played by the frictional loss.

(2) DRM has a peak value of the damping performance on the magnetic attractive force.

(3) $F_{\text {ult }}$, the ultimate value of the magnetic attractive force which gives the peak value of $\eta$ of DRM, was measured to be about $9.1 \mathrm{kPa}$ in DRM of 55 vol\% and $9.9 \mathrm{kPa}$ in DRM of 60 vol\%.

(4) The damping performance of DRM improves as the ferrite content decreases.

(5) The damping performance of DRM increases linearly with an increase of the vibratory amplitude of vibrating body.

(6) As the result of our experiments the damping performance of DRM is estimated by the following equations. 


$$
\begin{gathered}
\eta=\eta_{\mathrm{f}}+\eta_{\mathrm{i}} \\
\eta_{\mathrm{f}}=(r \times A+s) \times \mu(V) \times F \\
\eta_{\mathrm{i}}=(-c \times V+d) \times F \quad\left(F \leqq F_{\mathrm{ult}}\right) \\
\eta_{\mathrm{f}}=\left(r_{1} \times A+s_{1}\right) \times\left[\mu(V) \times \Delta F-s_{2} \times \mu(V)^{2} \times \Delta F\right. \\
\left.-s_{3} \times(\mu(V) \times \Delta F)^{2}\right] \\
+\left(r_{2} \times A+s_{4}\right) \times \mu(V)+r_{3} \times A+s_{6} \\
\eta_{\mathrm{i}}=\eta_{\mathrm{ic}} \quad\left(F \geqq F_{\text {ult }}\right)
\end{gathered}
$$

Where $\eta$ is the damping performance of DRM, $\eta_{\mathrm{f}}$ is the frictional loss, $\eta_{1}$ is the internal loss, $\eta_{\text {ic }}$ is the convergent value of the internal loss, $F$ is the magnetic attractive force per unit area $(\mathrm{kPa}), V$ is the ferrite content (vol\%), $A$ is the vibratory amplitude of vibrating body (m), $\Delta F$ is $F-F_{\text {ult }}$ (kPa), $r, s$, $c, d, r_{1}, r_{2}, r_{3}, s_{1}, s_{2}, s_{3}, s_{4}, s_{5}$ are constants. $r$ is about $3.0 \times 10^{4}(1 / \mathrm{m} / \mathrm{kPa}), s$ is about $2.9 \times 10^{-2}(1 /$ $\mathrm{kPa}), c$ is about $4.2 \times 10^{-4}(1 / \mathrm{kPa} / \mathrm{vol} \%), d$ is about $2.9 \times 10^{-2}(1 / \mathrm{kPa}), r_{1}$ is about $5.5 \times 10^{2}(1 / \mathrm{m} / \mathrm{kPa})$, $r_{2}$ is about $1.4 \times 10^{5}(1 / \mathrm{m}), r_{3}$ is about $2.7 \times 10^{4}(1 /$ $\mathrm{m}), s_{1}$ is about $5.3 \times 10^{-4}(1 / \mathrm{kPa}), s_{2}$ is about $5.1, s_{3}$ is about $1.1, s_{4}$ is about $1.3 \times 10^{-1}$ and $s_{5}$ is about $2.6 \times 10^{-2}$.

These formulations are applied to the following type of DRM, that is, the magnetic rubber layer is made of butyl rubber whose ferrite content is $40-60$ vol\% and thickness is about $3 \mathrm{~mm}$, the constraining layer is made of hydrated calcium silicate whose thickness is about $14 \mathrm{~mm}$, and the mass is about 65 g. The applicable frequency is about $400 \mathrm{~Hz}$. We are planning to do the following works to make clear the damping properties of DRM, one is to verify these formulations shown in this paper through more elaborate experiments with a large number of measuring data, and another is to generalize it through experiments with various kinds of samples and several frequencies other than that of this paper, and so on.

\section{ACKNOWLEDGMENTS}

We appreciate the cooperation of NICHIAS
Corporation and C.I. KASEI Co. Ltd., for the development of the Damping Material Applying Rubber Compounding Magnetic Powder (DRM).

\section{REFERENCES}

1) D. Johnson, "Design of passive damping systems," Trans. ASME 117, 171-176 (1995).

2) P. M. Morse, Vibration and Sound (McGraw-Hill Book Company, Inc., New York, 1948), p. 151.

3) M. Hansaka, "Damping properties of magnetic vibration damper," Inter-Noise 93, Vol. 2, 859-862 (1993).

4) M. Hansaka, "Damping properties of magnetic vibration damper,” Jpn. Railw. Eng. 126, 19-20 (1993).

5) M. Hansaka and N. Mifune, "Damping properties of magnetic vibration damper (MVP)," Inter-Noise '94, 4, 993-996 (1994).

6) M. Hansaka and N. Mifune, "Development of a new type high grade damper; magnetic vibration damper," Q. Rep. Railw. Tech. Res. Inst. Jpn. 35(3), 199-201 (1994).

7) M. Masako, Y. Ito, and C. Fujimoto, "Behavior of the horizontal stiffness and the micro-sliding on the bolded joint under the normal pre-load," Proc. 12th Int. M.T.D.R. Conf., 81-88 (1972).

8) E. M. Kerwin, Jr., "Damping of flexural waves by a constrained viscoelastic layer," J. Acoust. Soc. Am. 31, 952-962 (1959).

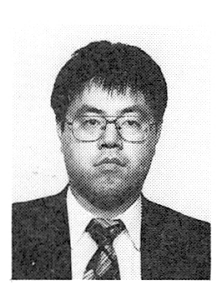

Masanori Hansaka An engineer of Organic Material Group belonging to Materials Technology Development Division of Railway Technical Research Institute of Japan. Mainly have been engaged in research and development of materials effective for vibration and noise control and improvement of application technology of these materials.

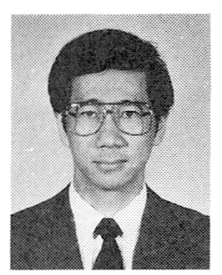

Naoto Mifune A chief engineer of Organic Material Group belonging to Materials Technology Development Division of Railway Technical Research Institute of Japan. Engaging in research and development of organic materials for railway extensively. In these works, the development of materials effective for vibration and noise control is paid attention. 